\title{
Factores que afectan al comportamiento de los pasajeros y a sus actividades en las terminales aeroportuarias
}

\section{Factors affecting passenger activity behavior in the airport terminal}

Núm. 9 (2017), pp. 5-25

Kalakou, Sofia *

Moura, Filipe **

García-Melero, Gustavo ***

Recibido: octubre, 2016

Aceptado: diciembre, 2017

JEL Clasif: Co1, C25, C51, D12, L93

DOI: $10.5944 /$ reppp.9.2017.20084

SOFIA KaLAKOU: Associate en VTM-Consultores \& Investigador en CERIS Group en Instituto Superior Técnico, Universidad de Lisboa, CESUR, DeCIVIL, Av. Rovisco Pais, 1, 1049-001, Lisboa, Portugal. E-mail: sophia.kalakou@vtm-global.com

** Filipe Moura: Profesor en Instituto Superior Técnico, Universidad de Lisboa, CESUR, DeCIVIL, Av. Rovisco Pais, 1, 1049-001, Lisboa, Portugal. E-mail: fmoura@tecnico.ulisboa.pt

** Gustavo García-Melero: Profesor en Pontificia Universidad Católica de Valparaíso, Escuela de Ingeniería de Transporte, Avenida Brasil, 2147, Valparaíso, Chile. E-mail: gustavo.garcia@pucv.cl 
Factores que afectan al comportamiento de los pasajeros y a

sus actividades en las terminales aeroportuarias

\title{
Resumen
}

En los últimos años el número de pasajeros está creciendo continuamente en el sector aéreo y no se prevé que esta tendencia disminuya en el futuro. El comportamiento de los pasajeros en las terminales aeroportuarias ha sido poco explorado en la literatura, a pesar de la relevancia de su estudio. El objetivo de este trabajo es analizar los patrones de comportamiento en la llegada de los pasajeros al Aeropuerto Internacional de Lisboa, identificando los factores que les afectan, a través de la modelización de las llegadas de pasajeros a la terminal mediante modelos de elección discreta. Los resultados indican que la hora del vuelo, el lugar de residencia, el modo de acceso a la terminal, el destino del vuelo y la frecuencia de viaje inciden sobre los patrones de llegada a la terminal respecto a la apertura de los mostradores de facturación. Estos resultados son relevantes para las configuraciones futuras de la terminal aeroportuaria.

Palabras clave: Aeropuerto; Modelos de elección discreta; Lisboa; Portugal

\begin{abstract}
In recent years the number of passengers is continuously growing in the air sector and this trend is not expected to decline in the future. The passengers' behavior in the airport terminals has not been explored deeply in the literature, despite the relevance of their study. The aim of this work is to analyze the arrival patterns of passengers in the Lisbon International Airport, through the modelling of passenger arrivals to the terminal using discrete choice models. The results indicate that flight departure time, place of residence, mode of access to the terminal, flight destination and frequency of travel affect the arrival patterns to the terminal regarding the opening of the check-in counters. These results are relevant for future configurations of the airport terminal.
\end{abstract}

Key Words: Airport; Discrete Choice Models; Lisbon; Portugal

Revista de Evaluación de Programas y Políticas Públicas | Núm. 9 (2017), pp. 5-25 


\section{Introducción}

Cada aeropuerto cumple con una amplia variedad de funciones y atiende a una gran diversidad de usuarios. Se pueden identificar tres grandes categorías de actividades aeroportuarias: servicios operativos esenciales, servicios de gestión del tráfico y servicios comerciales (Doganis, 1992). Los aeropuertos ofrecen una amplia variedad de servicios relacionados tanto con la preparación de los pasajeros para su viaje, como con su tiempo libre dentro de la terminal. En una terminal aeroportuaria se procesa el registro de los pasajeros y del equipaje, se conectan los pasajeros y el equipaje a las aeronaves, se transporta a los pasajeros entre vuelos, se acomodan los movimientos de las aeronaves y se provee espacio para áreas comerciales que ayudan a financiar el aeropuerto, entre otros.

Las instalaciones comerciales más comunes, no aeronáuticas, que se ubican en una terminal aeroportuaria son las tiendas libres de impuestos, las de regalos y prensa, las de alimentos y bebidas, las de productos especializados, así como los bares y restaurantes, que en conjunto satisfacen las necesidades más básicas de los pasajeros. En muchos aeropuertos se ofrecen actividades más especializadas (por ejemplo, centros de convenciones, hoteles, casinos, instalaciones recreativas), cuyo número y variedad han aumentado considerablemente en los últimos años, especialmente en las terminales con operaciones internacionales.

Aunque en muchos aeropuertos se pueden encontrar una amplia gama de actividades, no todos los pasajeros participan en todas ellas ni en las mismas actividades. El uso de estas áreas comerciales depende de factores que han sido poco explorados en la literatura. Obviamente, las preferencias de los pasajeros pueden diferir según sus características y las del propio aeropuerto, pero un factor determinante en la realización de actividades es el tiempo que los pasajeros tienen disponible en la terminal. Es de esperar que cuanto antes lleguen los pasajeros, más probable es que realicen actividades no aeronáuticas, pero el tiempo que los pasajeros pasan en los aeropuertos varía enormemente.

La complejidad en la vida de los aeropuertos se ha incrementado con el creciente número de pasajeros de los últimos años, y aún más lo hará en el futuro con las previsiones existentes (Banco Mundial, 2017). Para el año 2027 se prevé que los aeropuertos de todo el mundo manejarán 11.000 millones de pasajeros, dejando un déficit de capacidad de 1.000 millones de pasajeros (Airport World, 2015).

De acuerdo con la International Air Transport Association (IATA, 2013), alrededor de 3 mil millones de personas utilizaron el transporte aéreo por necesidades empresariales y turísticas en 2013, y según la World Tourism Organisation (WTO, 2014), alrededor del $51 \%$ de los turistas internacionales eligieron el avión para su transporte. La demanda aérea crece continuamente y está creando presión sobre los aeropuertos, los cuales deben asegurar que su capacidad se adapta adecuadamente tanto a las aeronaves como a los pasajeros. En este contexto, el papel de los pasajeros en las operaciones de los aeropuertos ha cambiado, ya que ahora son vistos como los clientes directos del aeropuerto, lo cual se aleja de la visión conservadora y anticuada de ser solamente clientes de la línea aérea (Graham, 2014). Por lo tanto, el incremento de los volúmenes de tráfico aéreo conlleva la necesidad de comprender mejor el comportamiento de los pasajeros, lo que supone un gran desafío ya que estamos ante una población cambiante. La demanda ha ido aumentando, pero algunos autores (Alegre, Mateo \& Pou, 2009) sostienen que

Revista de Evaluación de Programas y Políticas Públicas | Núm. 9 (2017), pp. 5-25 
Factores que afectan al comportamiento de los pasajeros y a

sus actividades en las terminales aeroportuarias

esto se atribuye a incrementos en la frecuencia de viaje de los pasajeros más que a un aumento real en el número de personas que viajan. Este argumento se puede aplicar especialmente al turismo moderno, que se caracteriza por estancias breves y frecuentes repartidas a lo largo del año (Salmasi, Celidoni \& Procidano, 2012; Ferrer-Rosell, Martínez-García \& Coenders, 2014), y para los viajes de negocios que cada vez son mayores (Barros \& Machado, 2010; Castillo-Manzano, Lopez-Valpuesta \& Gonzalez-Laxe, 2011). El efecto del envejecimiento de la población es otro de los elementos a considerar en la gestión de los aeropuertos, ya que se estima que para el año 2050 la población de 60 años o más, realizará más de 2.000 millones de viajes internacionales, mucho más que los 593 millones que realizaron en 1999 (Losada, Alén, Domínguez \& Nicolau, 2016).

La comprensión del comportamiento de los pasajeros sigue estando poco explorada, especialmente en el sector aéreo. Algunos estudios muestran información respecto a los tiempos de llegada de pasajeros en las terminales aeroportuarias. En particular, dicha información se presenta en estudios que pretenden simular procedimientos que involucran a los pasajeros, con el fin de evaluar el desempeño de los procesos de facturación, seguridad y equipaje. En este marco, se puede destacar el estudio realizado para simular los movimientos de los pasajeros y optimizar la asignación de los mostradores de facturación en el Aeropuerto Internacional de Seúl (Park \& Ahn, 2003), en el que se asumió:

- $\quad$ El $15 \%$ de los pasajeros de larga y corta distancia llegan 140 y 130 minutos, respectivamente, antes de la salida del vuelo al mostrador de facturación.

- $\quad \mathrm{El} 45$ \% de los pasajeros de larga y corta distancia llegan 90 y 60 minutos, respectivamente, antes de la salida del vuelo al mostrador de facturación.

- $\quad \mathrm{El} 40$ \% de los pasajeros de larga y corta distancia llegan 60 y 40 minutos, respectivamente, antes de la salida del vuelo al mostrador de facturación.

Los autores llevaron a cabo una encuesta para verificar sus supuestos y validaron que el número promedio obtenido, a partir de los comportamientos de llegada de los pasajeros de larga y corta distancia, coincidía con sus supuestos. En otros estudios en los que se evalúa la eficiencia operativa en el procesamiento de pasajeros (Van Boekhold, Faghri \& Li, 2014), se sostiene que estos llegan entre 1 y 4 horas antes de la salida del vuelo, utilizándose las indicaciones de International Air Transport Association (IATA, 2004) para la simulación de los tiempos de llegada de los pasajeros. Para la simulación de procesos en el Aeropuerto de Amsterdam (Joustra \& Van Dijk, 2001), se obtuvo que el $10 \%$ de los pasajeros llegan 240 minutos antes de la salida, el $25 \%$ entre 240 y 210 , el $25 \%$ entre 210 y 180 , el $20 \%$ entre 180 y 150 , el $7 \%$ entre 150 y 120 , el $10 \%$ entre 120 y 90 , y el $3 \%$ restante lo hacen cuando quedan menos de 90 minutos.

Otros estudios que han considerado el tiempo disponible de los pasajeros en las terminales aeroportuarias han analizado los factores impulsores del consumo y la generación de ingresos no aeronáuticos en los aeropuertos. En este contexto, cabe destacar el estudio realizado en el Aeropuerto de Oviedo (Torres, Domínguez, Valdés \& Aza, 2005) donde se obtuvo que los ingresos están correlacionados con el tamaño de la zona comercial disponible, así como con el tiempo que los pasajeros tienen disponible en la terminal antes de la salida del vuelo, pero, sin embargo, no demostraron que exista una relación entre la cantidad gastada y el tiempo disponible. 
Resultados similares se obtuvieron en el estudio realizado a partir de una muestra de 7 aeropuertos regionales españoles (Castillo-Manzano, 2010), donde se obtuvo que el tiempo de espera antes del embarque afectaba a la decisión del pasajero sobre sus consumos o compras en el área comercial de la terminal. En particular, se estimó la probabilidad de que un pasajero realice un gasto en función del tiempo de espera disponible en el aeropuerto: «si el tiempo de espera excede a una hora, hay un aumento del $10 \%$ en la probabilidad de consumir algún alimento o bebida y un aumento del $6 \%$ en la probabilidad de hacer una compra. Una vez que se ha tomado la decisión de gastar dinero, la cantidad aumentará con el tiempo. En un caso extremo, si el tiempo de espera excede de 3 horas, la probabilidad de que se consuman alimentos y bebidas y se realice una compra aumenta en $31 \%$ y $19 \%$, respectivamente, y la cantidad gastada aumenta en casi $41 \%$.

En un estudio realizado en el Aeropuerto de Taiwan (Lin \&Chen, 2013), se demostró que la presión del tiempo modera la relación entre las motivaciones comerciales y las actividades comerciales. En otros estudios (Tosic, 1992), se consideró que los viajeros de vacaciones y de ocio es probable que pasen más tiempo en la terminal en comparación con los viajeros de negocios por causa de niveles diferentes de presión de tiempo.

Los aeropuertos han desarrollado muchas actividades no aeronáuticas antes y después del control de seguridad, donde los pasajeros pueden ocupar su tiempo. Existen estudios (Ma y Yarlagadda, 2012), en los que se han empleado modelos basados en el agente (pasajero), incorporando las actividades no aeronáuticas como variables, que han llegado a clasificarlas en 10 categorías según sus propósitos (servicios de información, servicios monetarios, servicios de ayuda, relajación básica, conectividad social, autoservicio rápido, tiendas, declaración de impuestos y servicios relacionados con la religión). En estos estudios se estimaron las probabilidades condicionales de realizar cada actividad mediante el uso de redes bayesianas. Por otro lado, también existen otras clasificaciones de las actividades aeroportuarias no aeronáuticas (Popovic, Kraal \& Kirk, 2010): aquellas actividades opcionales relacionadas con el viaje, tales como el cambio de divisas, y aquellas actividades no relacionadas con el viaje como pueden ser las compras. En este mismo estudio también se identificaron 4 patrones de actividad: actividades en grupo, concurrentes, individuales y relacionadas con las pertenencias personales de los pasajeros.

Cada vez es mayor la complejidad de las características de los pasajeros, lo cual lleva a que los gestores de aeropuertos quieran comprender mejor la variedad de pasajeros existente, planifiquen sus instalaciones para ajustarlas a sus necesidades y motivaciones y asignen adecuadamente el espacio disponible (Graham, 2014). Los factores psicológicos han sido investigados en el contexto del comercio minorista, ya que la planificación previa de las actividades que los pasajeros realizan dentro del aeropuerto podría estar relacionada con el uso de estas áreas. Se ha encontrado que el gasto minorista está positivamente relacionado con el exceso de disponibilidad de tiempo (Torres et al., 2005; Castillo-Manzano, 2010; Bohl, 2014). Por otro lado, el concepto de «curva de tensión de viaje» refleja los cambios en los niveles de estrés durante el tiempo previo a la salida del vuelo; el período entre el control de inmigración y el control de seguridad en la puerta de embarque es el período menos estresante y genera oportunidades para actividades no aeronáuticas. En el mismo estudio, los pasajeros indicaron que eran más propensos a comprar después de pasar por el control del aeropuerto que antes; estar en un aeropuerto no es una actividad diaria cotidiana y, de hecho, aumenta los niveles de estrés de 
Factores que afectan al comportamiento de los pasajeros y a

sus actividades en las terminales aeroportuarias

los pasajeros. Este período se conoce como la «hora feliz», lo que sugiere que las compras en el aeropuerto se tratan de manipular en esta «hora feliz». Teniendo en cuenta las largas distancias que los pasajeros tienen que cubrir en la terminal del aeropuerto, esta «hora feliz» puede ser explotada de manera eficiente y ofrecer a los pasajeros un ambiente agradable en el que pueden pasar su tiempo antes de abordar (Volkova, 2009).

En definitiva, las investigaciones realizadas hasta la fecha muestran que el tiempo disponible en el aeropuerto puede afectar las actividades y los ingresos no aeronáuticos. Asimismo, el tiempo disponible en la terminal del aeropuerto está en función de la hora de llegada del pasajero y del tiempo necesario para realizar las actividades aeronáuticas, es decir, la facturación, el control de seguridad, aduanas e inmigración. En este estudio analizaremos los patrones de comportamiento en la llegada de los pasajeros al Aeropuerto Internacional de Lisboa, e identificaremos los factores que les afectan.

\section{Marco teórico en la modelización de preferencias de las actividades de pasajeros}

En este apartado se presenta el marco teórico empleado para la modelización de las preferencias de los pasajeros respecto a sus actividades, que fundamentalmente se basa en los Modelos de Elección Discreta (MED). Los modelos desagregados de demanda (o MED) tienen su fundamento en las elecciones observadas a nivel individual, y consideran que los individuos toman decisiones en forma racional, es decir, maximizando su utilidad individual. Las probabilidades de elección dependen del atractivo de cada alternativa y de las características socioeconómicas de los individuos.

Los MED consideran a una población de individuos que actúan de manera racional en la maximización de su utilidad neta personal -homo economicus- sujetos a restricciones ambientales, legales, sociales, físicas o presupuestarias. De este modo, el conjunto de alternativas sobre las cuales el individuo debe escoger está predeterminado, es decir, el efecto de las restricciones ya se ha tenido en cuenta, no afecta ni en la determinación del conjunto de alternativas disponibles, ni en el proceso de selección de la opción más conveniente. Los MED se utilizan para modelizar la elección de un decisor sobre un conjunto definido de alternativas. Su objetivo es utilizar los atributos de las diferentes alternativas, las características del tomador de decisiones o las interacciones de estos factores para explicar la elección del tomador de decisiones.

La teoría de la utilidad aleatoria supone que existe un cierto conjunto $A=\left\{A_{1}, \ldots . ., A_{n}\right\}$ correspondiente a las alternativas disponibles para los individuos de la población. Para un individuo en particular, el conjunto de alternativas disponibles " $q$ " es: $A(q) \in \mathrm{A}$. Además, existe un conjunto $X$ de vectores de características de los individuos y atributos, que se pueden medir para cada individuo.

Debido a que el investigador no posee información completa existen situaciones en las cuales dos individuos, aparentemente idénticos y sometidos al mismo conjunto de alternativas, se comportan de manera diferente. Un individuo puede elegir una opción que no es la que otorga mayor utilidad según lo estimó el modelo, por lo que se agrega un término estocástico $\left(\varepsilon_{i q}\right)$ a la función de utilidad, que captura los errores del modelo procedentes de varias fuentes posibles: atributos alternativos no observados, características 
individuales no observadas, errores de medición o variables proxy (Ben-Akiva y Lerman, 1985), quedando de la siguiente forma:

$$
U_{i q}=V_{i q}+\mathcal{E}_{i q}
$$

donde

$$
V_{i q}=\sum_{k=1}^{K} \theta_{i q} X_{i k q}
$$

De esta forma, un individuo $q$ escogerá $A_{i}$, si y solo si:

$$
U_{i q} \geq U_{j q}, \forall A_{i} \in A(q)
$$

Como se desconocen los términos estocásticos, se debe especificar una probabilidad de elección de $A_{i}$ :

$$
P_{i q}=\operatorname{prob}\left\{\varepsilon_{i q} \geq \varepsilon_{j q}+V_{j q}-V_{i q}, \forall A_{i} \in A(q)\right\}
$$

Pueden utilizarse diferentes tipos de variables: genéricas para todas las alternativas, específicas para algunas de las alternativas, o socioeconómicas relacionadas con las características de los tomadores de decisiones.

\section{Aeropuerto Internacional de Lisboa}

El Aeropuerto Internacional de Lisboa, conocido como Aeropuerto de Portela y desde Mayo de 2016 denominado como Aeropuerto Humberto Delgado, es el más grande de Portugal. Está situado a 7 kilómetros al norte de Lisboa y está conectado a las redes de metro y autobús público, lo que facilita su conectividad.

En 2015 el número total de pasajeros atendidos era de alrededor de 20 millones, dispone de conexiones con más de 130 destinos y operan más de 40 aerolíneas.

Cuenta con dos pistas de aterrizaje y dos terminales de pasajeros, que están comunicadas entre sí mediante un servicio gratuito de autobús. En la terminal 1 se operan los vuelos internacionales y en la terminal 2 los vuelos de las compañías lowcost.

Existen diferentes actividades discrecionales en las terminales, los pasajeros pueden disponer de servicios para alquiler de coches, cambio de divisas, consigna de equipaje, tiendas libres de impuestos, etc.

El alcance de este estudio ha llevado a que el trabajo de campo se haya desarrollado en la zona de la terminal dedicada a las salidas de vuelos, siendo en esta zona donde se realiza la facturación y el control de seguridad. 


\section{Determinación de los factores que afectan al comportamiento de los pasajeros}

Se realizó una encuesta de preferencias reveladas para recopilar información sobre las actividades de los pasajeros dentro de la terminal, desde su llegada al aeropuerto hasta el paso del área de seguridad. Los pasajeros que participan en programas especiales de viajero frecuente fueron excluidos, ya que fueron atendidos en controles de seguridad separados. Asimismo, se excluyó a los pasajeros de bajo coste porque son atendidos en una terminal exclusiva de bajo coste. Los pasajeros con movilidad restringida y los pasajeros que viajan con bebés también fueron excluidos, ya que utilizan zonas exclusivas. La recopilación de datos tuvo lugar durante la primera semana de Marzo de 2014 de 10 a 21 horas, fue aleatoria ( citados aleatoriamente para participar en uno de los siguientes pasos de su experiencia de viaje (antes del control de seguridad):

- $\quad$ En los lugares de actividades discrecionales,

- $\quad$ mientras camina alrededor, y

- $\quad$ en el área de control de seguridad.

A todos los pasajeros que participaron en la encuesta se les hicieron preguntas relacionadas con las áreas mencionadas y también se requirió su itinerario en la zona existente antes del control de seguridad. Los pasajeros de último minuto no fueron capturados adecuadamente en la encuesta. Se recogieron 529 respuestas válidas completas de las cuales 86 eran pasajeros en tránsito. Del total de encuestas realizadas 47 \% corresponden a viajeros que llegaron antes y $53 \%$ a viajeros que llegaron después de la apertura de la facturación.

El comportamiento de los pasajeros está influenciado por aspectos relacionados con el tiempo, sus características sociodemográficas personales, los detalles del viaje, sus planes de actividad y la percepción que tienen sobre la configuración del edificio. Por lo tanto, la encuesta se estructuró en 5 partes que se referían a las áreas antes mencionadas:

1. Tiempo: Se solicitó a los pasajeros que proporcionaran el horario de salida del vuelo y con cuánto tiempo de antelación llegaron al aeropuerto, el tiempo empleado en la facturación (si era necesaria), la hora a la que planearon llegar o llegaron al área de seguridad y cuándo les gustaría llegar a su puerta de embarque. Este tipo de información proporcionó conocimiento sobre las preferencias de tiempo de los pasajeros y el tiempo de riesgo que deciden tomar.

2. Información personal: Para poder relacionar las elecciones de los pasajeros con los tipos de pasajeros, se recopiló información personal: edad, sexo, propósito del viaje, nacionalidad, ciudad de residencia, frecuencia del viaje aéreo, estrés por vuelo, estrés por el tiempo y familiaridad con el edificio del aeropuerto.

3. Información sobre el viaje aéreo: La información pertinente sobre el viaje aéreo se refiere a los siguientes elementos: línea aérea, destino, número de equipajes, modo de facturación y modo de llegada al aeropuerto. Además, se solicitó a los pasajeros que informaran del número de pasajeros con los que viajaban, el número de no viajeros con los que llegaron al aeropuerto y, en el caso de que llegaran en coche, si utilizaban el 
estacionamiento o no.

4. Actividades: Se solicitó a los pasajeros reportar las actividades que realizaron dentro del aeropuerto desde que llegaron a la terminal hasta llegar a la zona del control de seguridad. De este modo, se puede identificar los principales atractivos que hacen que los pasajeros se desvíen de su próximo paso (control de seguridad).

5. Orientación: Se recogieron las cuestiones pertinentes a la facilidad para moverse dentro del edificio. Se les pidió a los pasajeros que recordasen si usaban las pantallas de información de vuelo y la señalización, si se perdían dentro del edificio y si usaban algún punto de referencia. Finalmente, atribuyeron al edificio un valor como un indicador de evaluación para la orientación.

A continuación, se presentan distintas figuras y tablas que recogen información estadística relacionada con la muestra recolectada, segmentada en dos grupos: pasajeros que llegan antes y después de la apertura de los mostradores de facturación de su vuelo. La Figura 1 muestra información personal sobre los pasajeros: si son hombres, si son portugueses, su frecuencia de viaje (menos de 3 veces al año (frec_1); 4-10 veces (frec_2); y más de 10 (frec_3)), si usan el aeropuerto por primera vez, si están familiarizados con la configuración del aeropuerto, si están estresados para llegar a su puerta, si tienen miedo de volar, si viven en la zona de Lisboa y si llegan solos al aeropuerto. Se observa un comportamiento similar de las variables asociadas al estrés, al miedo y a llegar solo al aeropuerto. Sin embargo, predominan entre los que llegan después del comienzo de la facturación aquellos que declaran ser hombres, portugueses, estar familiarizados y que residen en la zona de Lisboa. Respecto a la frecuencia de viaje, aquellos que viajan menos llegan antes y a medida que aumenta la frecuencia de viajes también se incrementa el número de pasajeros que llegan después. Finalmente, aquellos que viajan por primera vez llegan antes en un mayor porcentaje.

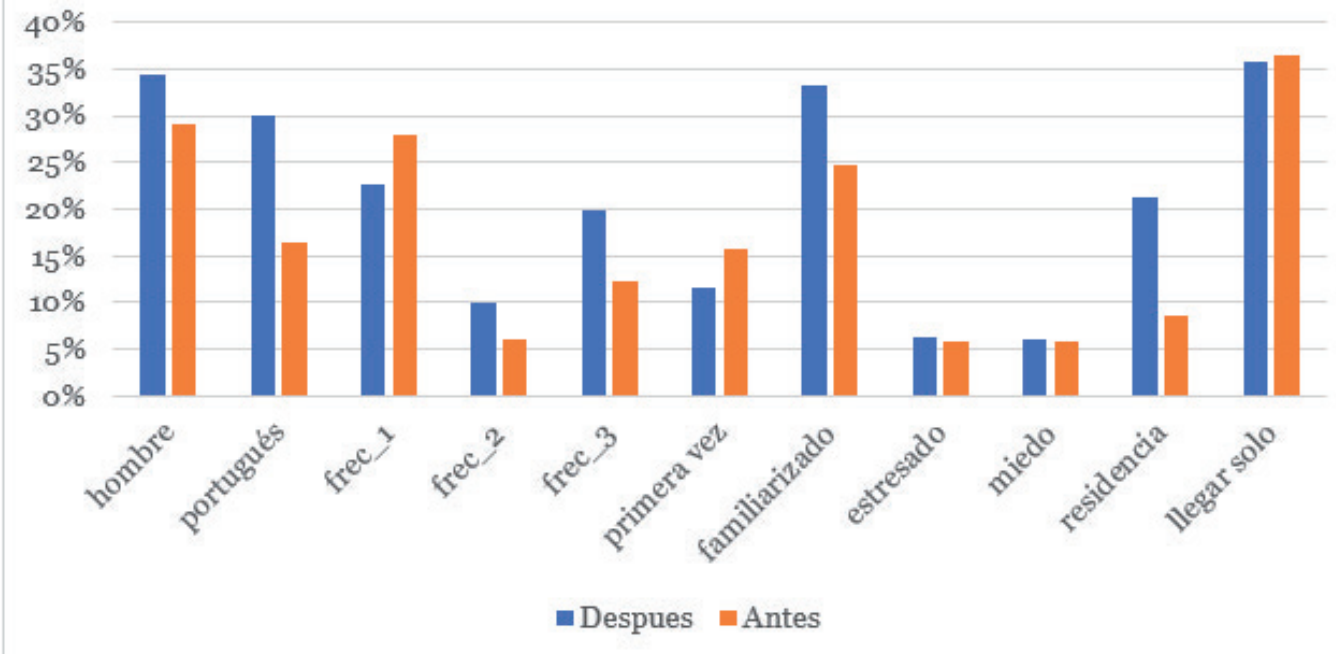

Figura I. Información personal 
Factores que afectan al comportamiento de los pasajeros y a sus actividades en las terminales aeroportuarias

Tabla I. Información personal.

\begin{tabular}{|l|c|c|c|}
\cline { 2 - 4 } \multicolumn{1}{c|}{} & Después & Antes & $\%$ sobre total muestra \\
\hline frec_I & $23 \%$ & $28 \%$ & $50,56 \%$ \\
\hline frec_2 & $10 \%$ & $6 \%$ & $16,03 \%$ \\
\hline frec_3 & $20 \%$ & $12 \%$ & $32,05 \%$ \\
\hline primer viaje & $1 \%$ & $1 \%$ & $1,35 \%$ \\
\hline hombre & $34 \%$ & $29 \%$ & $63 \%$ \\
\hline mujer & $19 \%$ & $18 \%$ & $37 \%$ \\
\hline portugués & $30 \%$ & $16 \%$ & $47 \%$ \\
\hline no portugés & $23 \%$ & $31 \%$ & $53 \%$ \\
\hline primera vez & $12 \%$ & $16 \%$ & $47 \%$ \\
\hline no primera vez & $23 \%$ & $31 \%$ & $53 \%$ \\
\hline familiarizado & $33 \%$ & $25 \%$ & $58 \%$ \\
\hline no familiarizado & $20 \%$ & $22 \%$ & $42 \%$ \\
\hline estresado & $6 \%$ & $6 \%$ & $12 \%$ \\
\hline no estresado & $41 \%$ & $47 \%$ & $88 \%$ \\
\hline miedo & $6 \%$ & $6 \%$ & $12 \%$ \\
\hline no miedo & $47 \%$ & $41 \%$ & $88 \%$ \\
\hline residencia & $21 \%$ & $9 \%$ & $30 \%$ \\
\hline no residencia & $32 \%$ & $38 \%$ & $70 \%$ \\
\hline Ilegar solo & $36 \%$ & $36 \%$ & $72 \%$ \\
\hline no llegar solo & $17 \%$ & $11 \%$ & $28 \%$ \\
\hline
\end{tabular}

En la Figura 2 vemos información relativa al vuelo como el propósito por el que viajaban (asuntos personales, business o turismo), si tenían equipaje para registrar y cómo se registraron (online, counter o machine), el tipo de destino del pasajero (Schengen, doméstico), si el viaje es al destino final, cuántos viajaron durante el fin de semana y en horas de la mañana antes de las $12 \mathrm{pm}$. Se observa un comportamiento similar para casi todas las variables, pero cabe destacar la dominancia que presenta el llegar después para los viajeros por negocios, los que viajan antes de las $12 \mathrm{pm}$ y aquellos que realizan la facturación online.

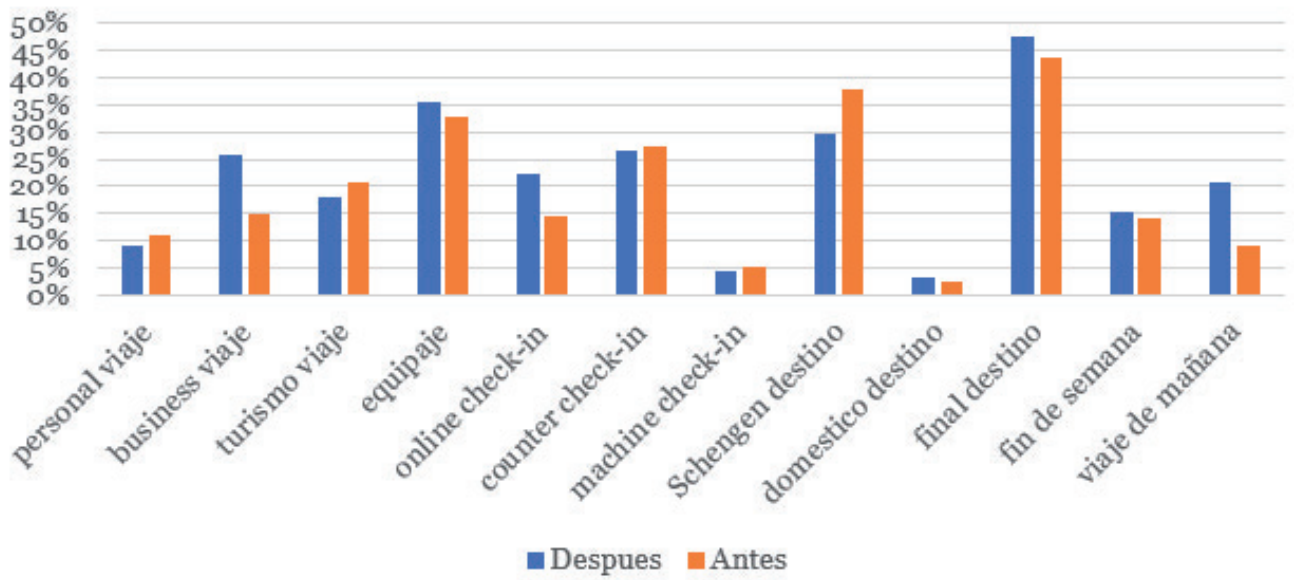

Figura 2. Información del vuelo. 
Tabla 2. Información del vuelo.

\begin{tabular}{|l|c|c|c|}
\cline { 2 - 4 } \multicolumn{1}{c|}{} & Después & Antes & $\%$ sobre total muestra \\
\hline personal & $9 \%$ & $11 \%$ & $20 \%$ \\
\hline business & $26 \%$ & $15 \%$ & $41 \%$ \\
\hline turismo & $18 \%$ & $21 \%$ & $39 \%$ \\
\hline online & $22 \%$ & $15 \%$ & $37 \%$ \\
\hline counter & $26 \%$ & $27 \%$ & $54 \%$ \\
\hline machine & $4 \%$ & $5 \%$ & $9 \%$ \\
\hline Schengen destino & $30 \%$ & $38 \%$ & $67 \%$ \\
\hline domestico destino & $3 \%$ & $3 \%$ & $6 \%$ \\
\hline international & $20 \%$ & $6 \%$ & $27 \%$ \\
\hline final destino & $47 \%$ & $44 \%$ & $91 \%$ \\
\hline no final destino & $6 \%$ & $3 \%$ & $9 \%$ \\
\hline fin de semana & $15 \%$ & $14 \%$ & $30 \%$ \\
\hline no fin de semana & $38 \%$ & $33 \%$ & $70 \%$ \\
\hline viaje de mañana & $21 \%$ & $9 \%$ & $30 \%$ \\
\hline no viaje de mañana & $32 \%$ & $38 \%$ & $70 \%$ \\
\hline equipaje & $36 \%$ & $33 \%$ & $69 \%$ \\
\hline no equipaje & $17 \%$ & $14 \%$ & $31 \%$ \\
\hline
\end{tabular}

La Figura 3 muestra las estadísticas sobre cuántos pasajeros habían planeado sus actividades antes de llegar al aeropuerto, cuántos ubicaron su puerta antes, cuántos pasajeros realizaron su embarque directamente después del control de seguridad, si se sintieron confundidos o perdidos y, cuántos pasajeros siguieron la señalización. El comportamiento es similar para todas las variables excepto para aquellos que han planeado sus actividades antes de llegar al aeropuerto, donde predominan los que llegan después.

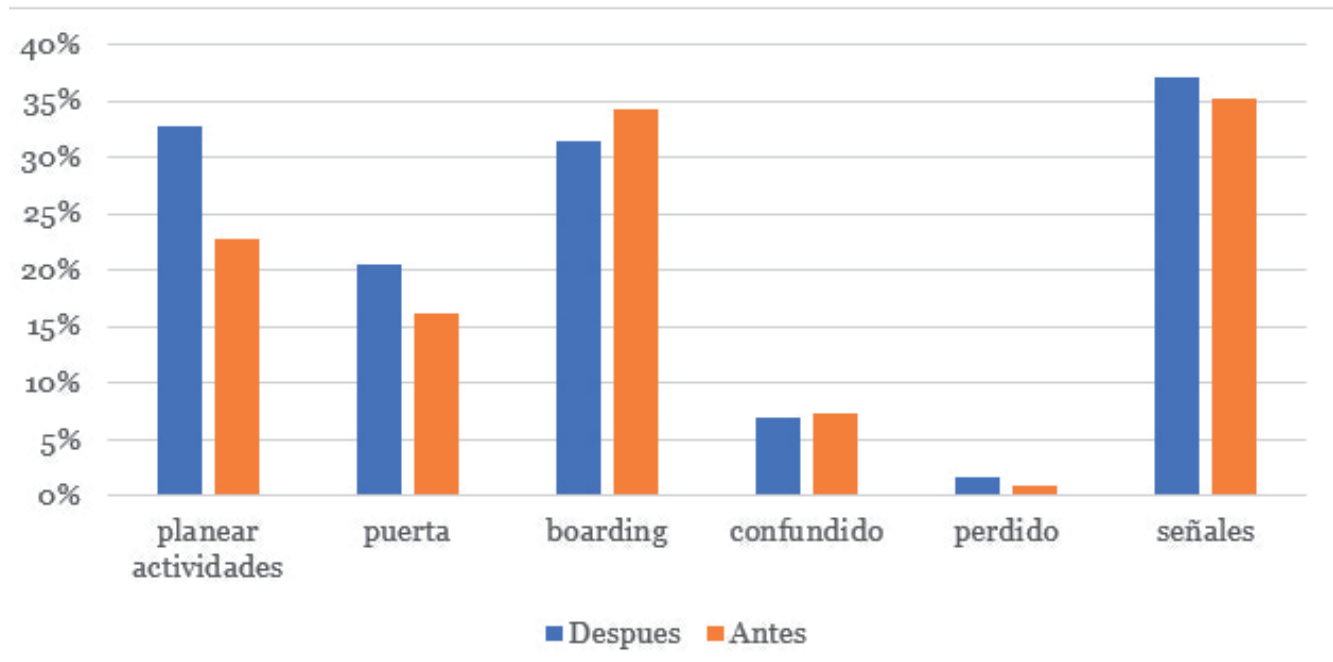

Figura 3. Información del comportamiento y percepción. 
Factores que afectan al comportamiento de los pasajeros y a sus actividades en las terminales aeroportuarias

Tabla 3. Información del comportamiento y percepción.

\begin{tabular}{|l|c|c|c|}
\cline { 2 - 4 } \multicolumn{1}{c|}{} & Después & Antes & $\%$ sobre total muestra \\
\hline planear actividades & $33 \%$ & $23 \%$ & $56 \%$ \\
\hline no planear actividades & $20 \%$ & $24 \%$ & $44 \%$ \\
\hline puerta & $21 \%$ & $16 \%$ & $37 \%$ \\
\hline no puerta & $32 \%$ & $31 \%$ & $63 \%$ \\
\hline boarding & $31 \%$ & $34 \%$ & $66 \%$ \\
\hline no boarding & $22 \%$ & $13 \%$ & $34 \%$ \\
\hline confundido & $7 \%$ & $7 \%$ & $14 \%$ \\
\hline no confundido & $46 \%$ & $40 \%$ & $86 \%$ \\
\hline perdido & $2 \%$ & $1 \%$ & $2 \%$ \\
\hline no perdido & $51 \%$ & $46 \%$ & $98 \%$ \\
\hline señales & $37 \%$ & $35 \%$ & $72 \%$ \\
\hline no señales & $16 \%$ & $12 \%$ & $28 \%$ \\
\hline
\end{tabular}

La Figura 4 presenta el modo de llegada al aeropuerto: taxi, autobús, metro, coche propio, coche alquilado y coche acompañante. Se observa que los pasajeros que acceden en taxi, en coche propio o en coche acompañante llegan después en mayor porcentaje, mientras que aquellos que llegan en autobús, metro o en coche alquilado lo hacen antes.

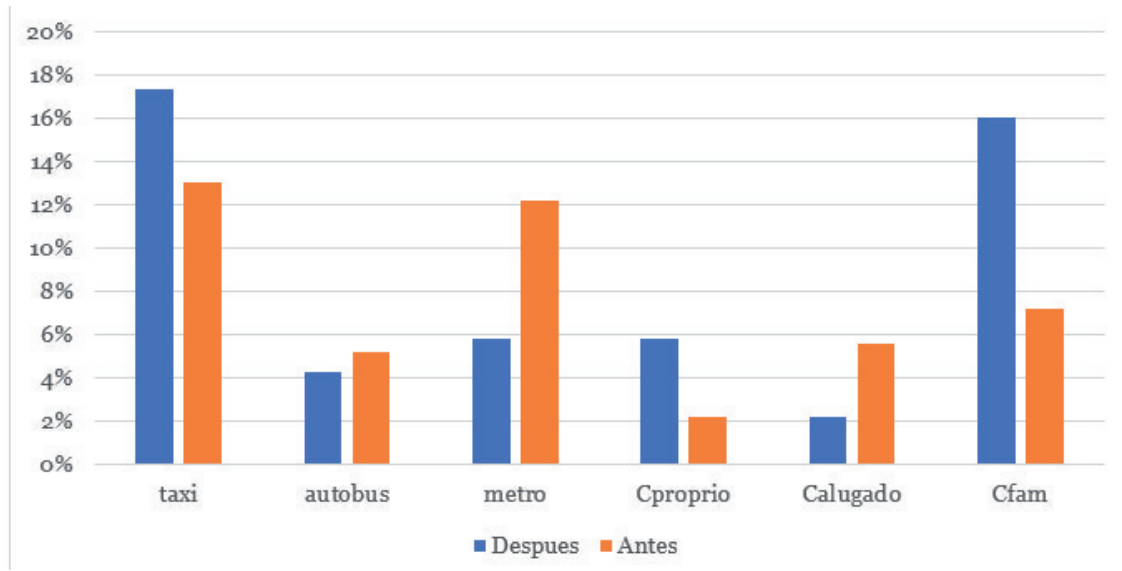

Figura 4. Información del modo de llegada.

Tabla 4. Información del modo de llegada.

\begin{tabular}{|l|c|c|}
\cline { 2 - 3 } \multicolumn{1}{c|}{} & Después & Antes \\
\hline taxi & $17 \%$ & $13 \%$ \\
\hline autobus & $4 \%$ & $5 \%$ \\
\hline metro & $6 \%$ & $12 \%$ \\
\hline Cproprio & $6 \%$ & $2 \%$ \\
\hline Calugado & $2 \%$ & $6 \%$ \\
\hline Cfam & $16 \%$ & $7 \%$ \\
\hline outro & $1 \%$ & $2 \%$ \\
\hline
\end{tabular}


Se calcularon las correlaciones entre todas las variables presentadas anteriormente y los resultados no mostraron correlaciones considerables $(>0,19)$ estadísticamente significativas. Para las variables binarias se calculó el coeficiente Phi y para las variables continuas se probaron los coeficientes de Pearson y Spearman. No es sorprendente que los valores más altos se hayan reportado para los pasajeros portugueses que estén familiarizados con el aeropuerto $(0,44)$. Además, observamos que existe correlación entre los pasajeros que son portugueses pero no viven en la zona de Lisboa $(-0,41)$, ya que Portela es el principal aeropuerto portugués. Por último, los pasajeros que llegan solos están inversamente correlacionados con llegar en coche $(-0,43)$.

La Figura 5 presenta el número de actividades no aeronáuticas realizadas por los pasajeros en su experiencia en la terminal, desde el momento en que llegaron al aeropuerto hasta llegar al control de seguridad. El 39 \% de los pasajeros no realizó ninguna actividad, el 44 \% realizó una actividad no aeronáutica, el $12 \% 2$ actividades, el $4 \% 3$ actividades y solo el $1 \%$ más de 3 .

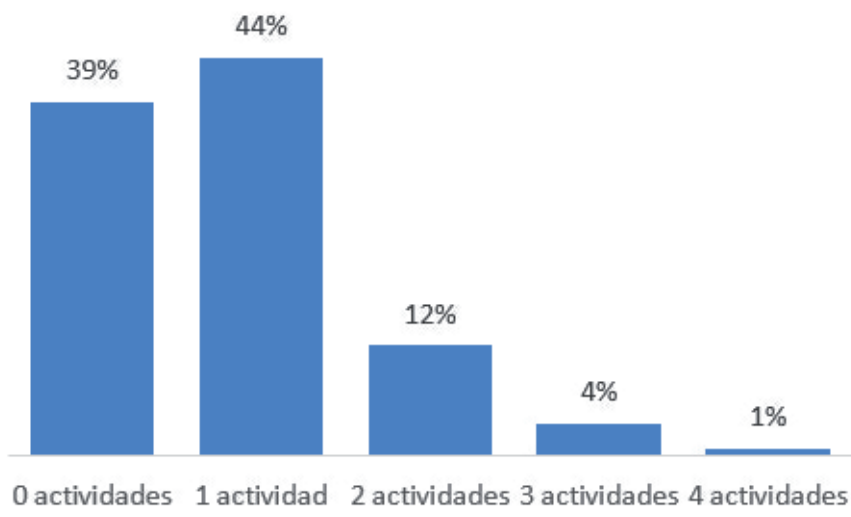

Figura 5. Información de las actividades.

Antes del control de seguridad, el 33,8 \% de los pasajeros restringía sus actividades a las tareas aeronáuticas relacionadas con su viaje. Casi uno de cada 8 pasajeros $(12,9 \%)$ fue directamente al control de seguridad después de llegar al aeropuerto. En general, el $47 \%$ de los pasajeros realizaba solo actividades aeronáuticas antes de la seguridad y el 53 \% optaba también por realizar actividades discrecionales. Después de la seguridad, la mayoría de los pasajeros dijeron que estaban planeando ir a tiendas y áreas de alimentación. La mayoría de los pasajeros (35,2 \%) utilizaron la zona de facturación y eligieron realizar una actividad discrecional que podría ser una visita a una zona de alimentación, una zona de venta al por menor o la zona de salón.

El patrón de llegada de pasajeros se presenta en la Figura 6 en intervalos de tiempo de 20 minutos. Se observa un pico en el período de tiempo 120-160 minutos antes de la salida del vuelo. En el Aeropuerto de Lisboa, en 2014 los mostradores de facturación estaban abiertos para el registro de pasajeros y equipajes 120 y 180 minutos antes de la salida de los vuelos a los destinos de Schengen y no Schengen, respectivamente. Una pequeña proporción de las líneas aéreas establecidas en el aeropuerto abren sus mostradores 240 minutos antes de las salidas de vuelo para los destinos en África. La Figura 6 muestra que las llegadas de pasajeros en el aeropuerto se vuelven más elevadas poco antes de la apertura de los mostradores de facturación de vuelos con destinos 
de Schengen. También se presenta que un pequeño porcentaje de pasajeros llega antes de las 4 horas antes de la salida de su vuelo. Teniendo en cuenta el hecho de que el Aeropuerto de Lisboa tiene conexiones directas desde el centro de la ciudad a través de un autobús y una línea de metro, y se encuentra a una distancia de $7 \mathrm{~km}$ del centro de la ciudad, se explica el hecho del pico de llegadas antes de la apertura de Schengen. Como el tráfico de pasajeros del aeropuerto ha estado aumentando durante los últimos 5 años, es probable que los pasajeros que viajan al aeropuerto se comporten impulsados por la incertidumbre de su hora de llegada al aeropuerto y la ansiedad de llegar a su puerta a tiempo y no perder el vuelo. El alto nivel de conectividad de los transportes al aeropuerto puede justificar las densas llegadas al margen del horario propuesto de llegada desde el punto de vista del aeropuerto, cercano a la apertura de los mostradores.

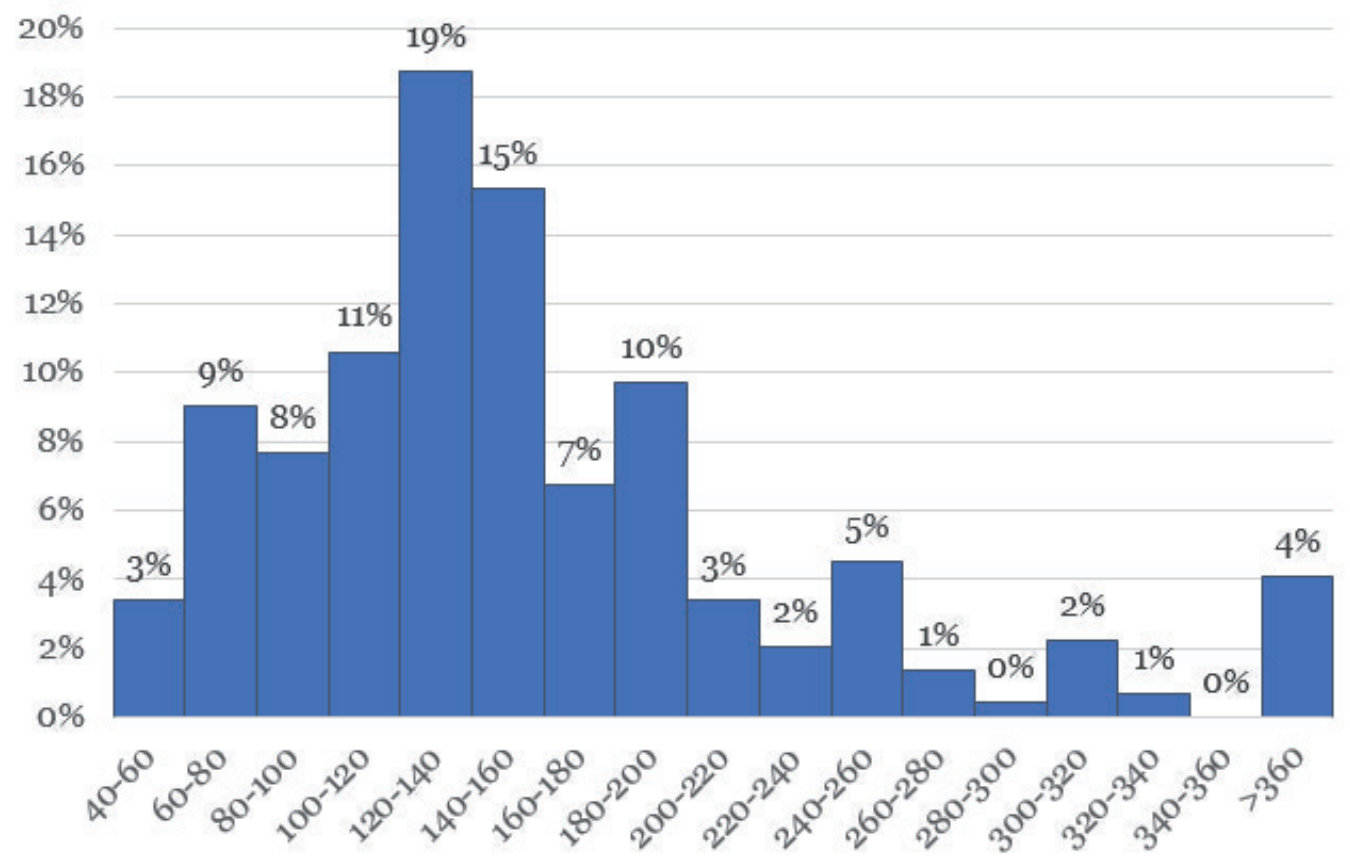

Figura 6. Patrón de llegada de los pasajeros.

En comparación con las normas internacionales y los patrones utilizados para propósitos de planificación, como los de la International Air Transport Association (IATA, 2004), se observa que nuestro patrón de llegada está sesgado hacia la derecha (más alejado de la llegada del vuelo), en aproximadamente 40 minutos. Esto puede tener implicaciones significativas para el uso del espacio del aeropuerto y el nivel de servicio proporcionado. Sin embargo, este patrón de llegada no difiere significativamente de los resultados que se encuentran en la literatura relativos a otros aeropuertos.

En la Figura 7 se puede observar los patrones de llegada de los pasajeros en base a si realizan 0, 1, 2 o 3 actividades no aeronáuticas antes del control de seguridad. Existe una tendencia a realizar más actividades a medida que los pasajeros llegan antes, mientras que la mayoría de los pasajeros que no realizan actividades llegan más tarde. Se observa un comportamiento similar cuando los pasajeros llegan cerca de la apertura de los vuelos Schengen. 


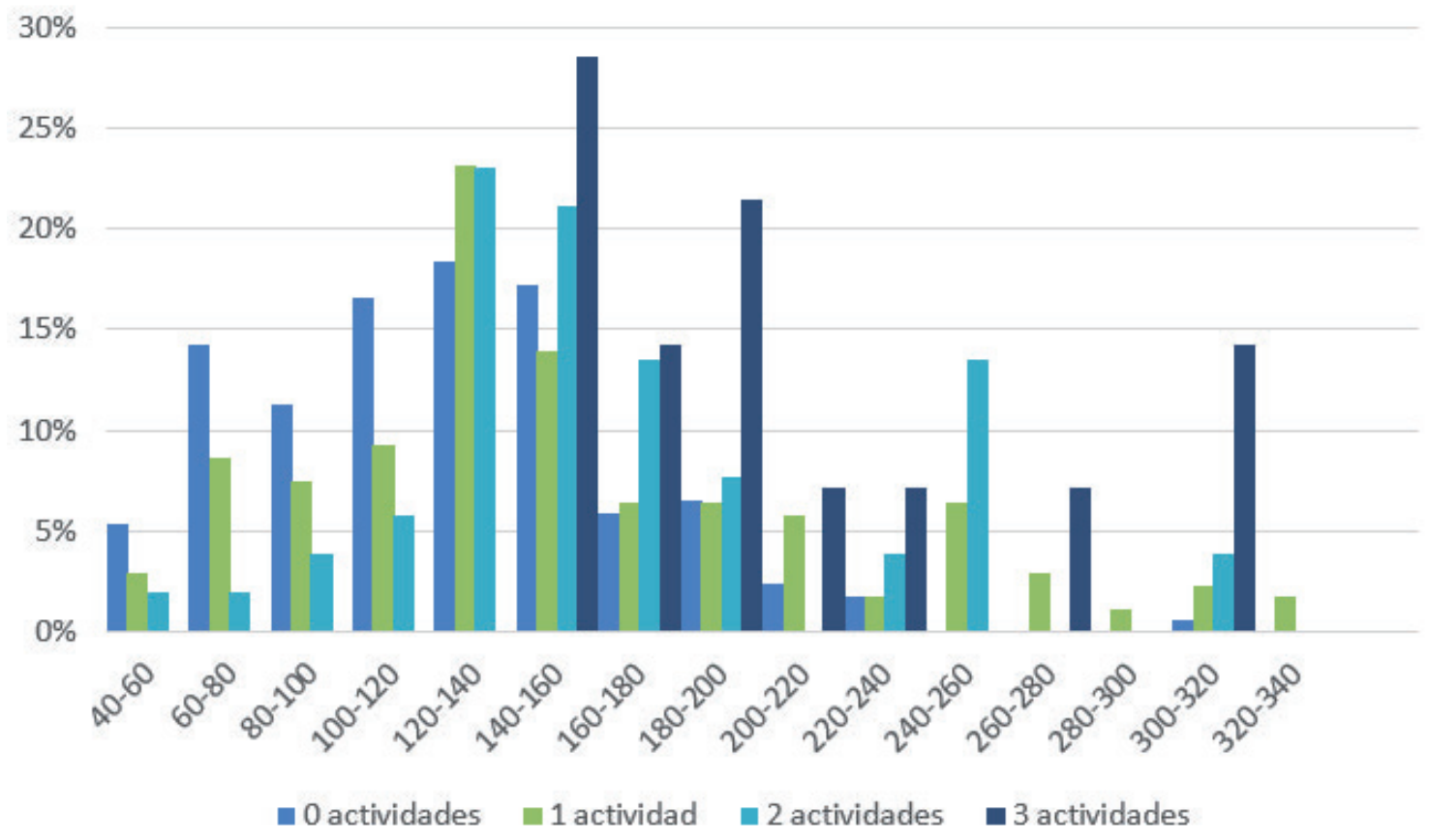

Figura 7. Patrón de llegada por grupo de actividad

En la siguiente sección, se trata de explicar los factores que afectan a la llegada de los pasajeros antes o después de la apertura de los mostradores de facturación de equipaje.

\section{Modelización de las llegadas de pasajeros a la terminal}

Las elecciones de los pasajeros fueron modeladas en SPSS. Inicialmente, intentamos estimar un modelo lineal con los tiempos reales de llegada de pasajeros medidos en minutos antes de la salida del vuelo. Para la estimación del modelo solo se utilizaron observaciones de pasajeros de salida; los pasajeros en tránsito fueron excluidos. Sin embargo, no se derivó una explicación clara del análisis. En consecuencia, modelizamos la llegada de los pasajeros con la apertura de la facturación de su vuelo como punto de referencia. Como hay límites en la salida de pasajeros (horarios de vuelo), el tiempo con que los pasajeros llegan al aeropuerto antes de la salida de su vuelo es un factor de planificación determinante; las llegadas tempranas de pasajeros implican una mayor concentración de pasajeros en la terminal. Un punto de referencia para las llegadas de pasajeros es la apertura de los mostradores de facturación dentro del aeropuerto. Por este motivo, la elección de los pasajeros para llegar al aeropuerto antes (alternativa «antes») o después (alternativa «después»), de la apertura de los mostradores de facturación, fue modelizada para analizar los factores que la afectan y luego explorar cómo las probabilidades de elección de llegada cambiarían al asumir cambios en las características de la población. La elección no fue dada como una respuesta del pasajero, pero fue calculada por los autores basándose en la hora de llegada y la salida del vuelo. 
Factores que afectan al comportamiento de los pasajeros y a

sus actividades en las terminales aeroportuarias

Dado que la naturaleza de la elección no depende de atributos específicos de las alternativas, sino más bien de la propensión a una acción desde el lado del viajero, solo se usaron características de pasajeros en la especificación del modelo. El modelo describe el efecto de las características de los pasajeros que inician su viaje (salidas) en sus preferencias para llegar antes o después de la apertura de los mostradores de facturación. Se debe señalar que la elección de llegar antes o después es la elección real de cada pasajero, este modelo no tiene en cuenta las intenciones previas de los pasajeros antes de llegar al aeropuerto. Existe la posibilidad de que los pasajeros tengan la intención de llegar más temprano o más tarde, pero el resultado final podría diferir debido a incertidumbres como retrasos inesperados, tráfico o tiempo de espera para transporte público, entre otros.

Los juicios a priori sobre cómo cada factor puede afectar a la llegada de pasajeros antes o después de la apertura de los mostradores de facturación se presentan a continuación, y la variable correspondiente utilizada en el modelo se indica al final de cada supuesto:

- $\quad$ Hora de vuelo: Se espera que los pasajeros que parten en la mañana lleguen después de la apertura de la facturación, ya que existe menos tiempo disponible para organizar todas sus actividades diarias antes de la salida (código de variable: «Mañana»).

- $\quad$ Residencia: Se espera que la probabilidad de llegar después de la apertura de los mostradores de facturación sea mayor para los residentes de la zona de Lisboa (código de variable: «Residencia»). Se supone que cuanto mayor sea la distancia a recorrer para llegar al aeropuerto, mayor será la incertidumbre de la hora de llegada. Por ejemplo, para los pasajeros que empiezan su viaje desde una ciudad cercana al aeropuerto, pero todavía necesitan usar una autopista, se espera que salgan de su destino antes para mitigar el riesgo de llegar tarde al aeropuerto debido a factores inesperados, por ejemplo. accidentes de tráfico y retrasos en la carretera, malas condiciones climáticas, daños mecánicos del modo de transporte (coche, tren, autobús, etc.), etc.

- $\quad$ Modo de llegada: Se espera que los pasajeros que lleguen en coche (código de variable: «Coche_Familiar») de un amigo o familia lleguen después de la apertura de los mostradores de facturación.

- $\quad$ Destino de viaje: Basado en la literatura previa y en los patrones observados, se espera que la utilidad de llegar antes de la apertura de los mostradores de facturación sea mayor para los pasajeros que viajan a destinos de Schengen (código variable: «Schengen»).

- $\quad$ Frecuencia de viaje: Se espera que cuanto más viaje un pasajero, más probable es que llegue después de la apertura de la facturación (código variable: «Alta_frequencia») debido a su familiaridad con los procesos del aeropuerto.

A menudo se observa que las constantes específicas de la alternativa (ASC), se agregan en la especificación de la función de utilidad de cada alternativa, para capturar el efecto promedio sobre la utilidad de todos los factores que no están incluidos en el modelo. Dado que hay solo dos alternativas - llegan antes (código: «antes») o después de la apertura de la facturación (código: «después») - la especificación de la alternativa «antes» incluye solo el ASC y la utilidad determinística es fijada a cero. El ASC equivalente 
se incluye en la utilidad de la alternativa «después» con los parámetros relacionados con todas las variables seleccionadas. Por lo tanto, las especificaciones de las utilidades que explican la llegada de los pasajeros antes y después de la apertura de la facturación son las siguientes:

$$
\begin{aligned}
& \mathrm{V}_{\text {después }}=\mathrm{ASC}_{\text {después }} \\
& \mathrm{V}_{\text {antes }}=\mathrm{ASC}_{\text {antes }}
\end{aligned}
$$

$$
\begin{aligned}
& +\beta_{\text {mañana }}{ }^{*} \text { Mañana } \\
& +\beta_{\text {resid }} * \text { Residencia } \\
& +\beta_{\text {Cfam }} * \text { Coche_Familiar } \\
& +\beta_{\text {Schengen }} * \text { Schengen } \\
& +\beta_{\text {frecuente }} * \text { Alta_frequencia }
\end{aligned}
$$

Con el fin de evaluar el impacto real de las variables en las elecciones de los indivi-

\begin{tabular}{|c|c|c|}
\hline Parámetro & Descripción de la variable asociada & $\begin{array}{c}\text { Valor } \\
\text { parámetro }\end{array}$ \\
\hline ASC_antes & & 2.021 \\
\hline$\beta_{\text {resid }}$ & I si el pasajero vive en Lisboa & $-0.886^{* * *}$ \\
\hline$\beta_{\text {mañana }}$ & I si el vuelo parte antes de las 12 de la mañana & $-0.889^{* * *}$ \\
\hline$\beta_{\text {frecuente }}$ & I si el pasajero viaja más de 10 veces por año & $-0.7^{* * *}$ \\
\hline$\beta_{\text {schengen }}$ & I si el pasajero viaja a un destino de la zona Schengen & $\mathrm{I} .156^{* * *}$ \\
\hline$\beta_{\text {Cfam }}$ & I si el pasajero llega al aeropuerto con un coche acompañante & $-0.646^{* * *}$ \\
\hline \multicolumn{2}{|c|}{ Número de observaciones } & 443 \\
\hline \multicolumn{2}{|c|}{ Log Likelihood $\left(L\left(\beta^{\prime}\right)\right)$} & 528.499 \\
\hline \multicolumn{2}{|c|}{ Hosmer and Lemeshow test } & $\begin{array}{l}10.849 \\
(0.093)\end{array}$ \\
\hline
\end{tabular}
duos, se pueden probar diferentes especificaciones. Para cada una de ellas, los parámetros se estiman por máxima verosimilitud, y se aplican pruebas estadísticas para evaluar la calidad de la especificación. En este caso, los signos atribuidos a los parámetros son consistentes con los supuestos a priori. Como no hay otro estudio pertinente, no podemos comparar nuestros hallazgos con estudios previos.

Tabla 5. Resultados del modelo estadístico.

No existen variables dominantes en nuestro modelo. La influencia de las variables estadísticamente significativas es similar, sobremanera para las que tienen signo negativo. Se encuentra que «Schengen» tiene un valor de parámetro más alto. Se probaron más variables para la especificación del modelo, pero no se demostró que contribuyesen a la mejora del modelo para explicar las elecciones de los pasajeros (género, día de la 
Factores que afectan al comportamiento de los pasajeros y a

sus actividades en las terminales aeroportuarias

semana, si el pasajero llega solo, el propósito del viaje, la edad, el equipaje, si el pasajero planificó las actividades que realizará en el aeropuerto, si el pasajero estaba familiarizado con la configuración del aeropuerto, si el pasajero partió por primera vez desde este aeropuerto, si viajó durante el fin de semana, con niños y varias interacciones de estas características). El valor de la prueba de Hosmer y Lemeshow muestra que el modelo es fiable para explicar las elecciones de los pasajeros.

A continuación, analizamos un escenario en el que los pasajeros que viajan frecuentemente aumentarán de una cuota del $32 \%$ al $35 \%, 38 \%$, 42 \% y $45 \%$ del total de viajeros. Con el fin de probar este escenario, hemos creado 10 nuevos conjuntos de datos para los cuales hemos cambiado aleatoriamente la alta frecuencia de o a 1. Las previsiones del modelo entregaron resultados estables y mostraron que la cuota de pasajeros que llegan antes de la apertura de la facturación al mostrador seguirá siendo el mismo hasta llegar al $45 \%$ de pasajeros cuando disminuye de $47 \%$ a $46 \%$ frente a esa variación.

Además, ponemos a prueba la sensibilidad del modelo respecto a las variaciones en el número de pasajeros que viven en la zona de Lisboa. Al disminuir este porcentaje de pasajeros de $30 \%$ a $27 \%, 24 \%, 21 \%$ y, finalmente $18 \%$, se produce un pequeño aumento en el número de pasajeros.

Teniendo en cuenta el efecto conjunto de estos posibles cambios en el futuro, observamos cambios marginales en el tiempo de llegada respecto a la apertura. La variación del porcentaje del número total de pasajeros prácticamente sigue siendo la misma, lo que indica que en el futuro las variaciones en los distintos perfiles de pasajeros del aeropuerto pueden dar lugar a resultados individualmente diferentes. Sin embargo, su efecto conjunto puede conducir a resultados holísticos estancados, como la continuación del patrón de llegada observado hoy en día.

Este cambio en las cuotas implica que el área de la terminal antes de la seguridad será menos utilizada a medida que aumenta la frecuencia de viaje. Como tal, los gerentes del aeropuerto podrían utilizar esta área redundante para actividades alternativas. Este análisis presenta la utilidad potencial de este modelo de comportamiento de los pasajeros, con el fin de estimar los requerimientos de área ante los cambios previsibles de las características de los viajeros.

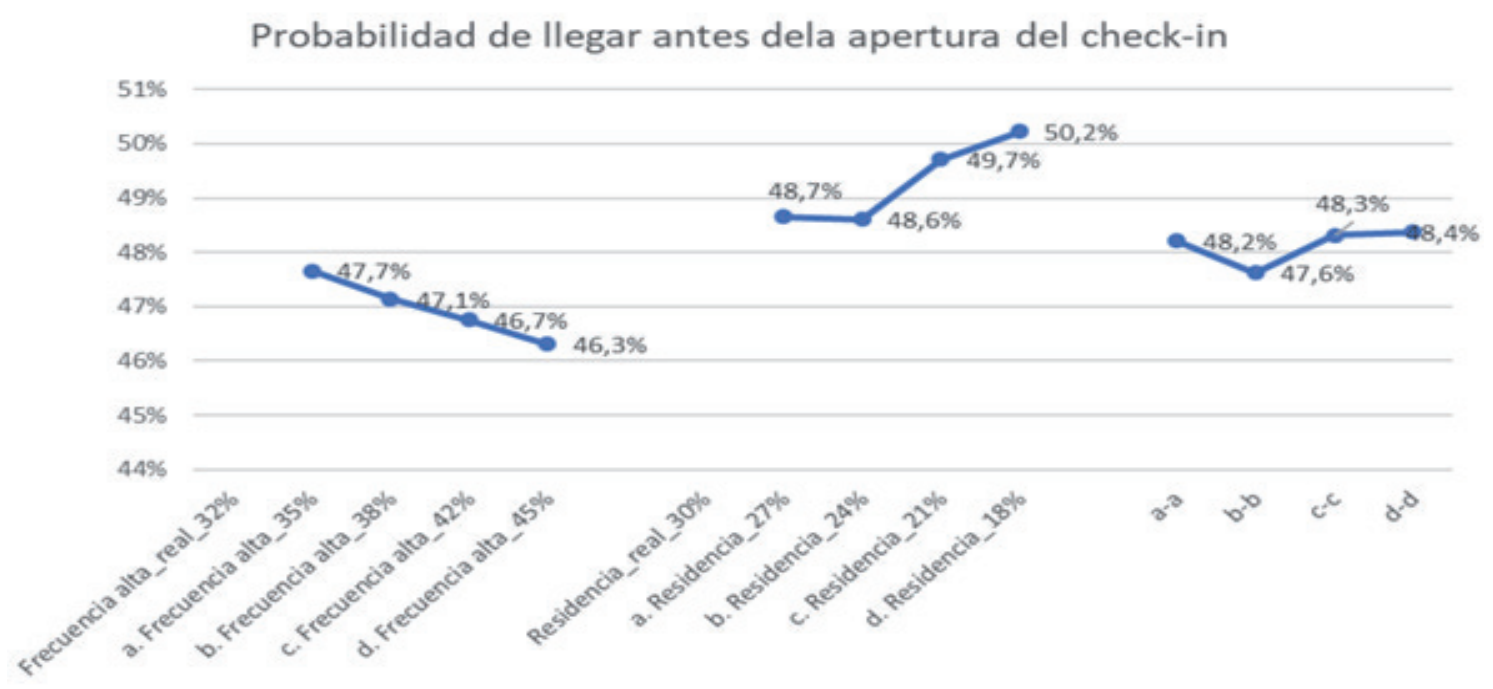

Figura 8. Probabilidad de llegar antes de la apertura de la facturación. 


\section{Conclusión}

Este trabajo se ha desarrollado en base a las llegadas de los pasajeros a la terminal del Aeropuerto de Lisboa. Se realizó una encuesta para recoger datos sobre el comportamiento de los pasajeros y se obtuvieron 529 respuestas. Se modelizaron las llegadas de pasajeros en relación con la apertura de los mostradores de facturación. Se constató que el área de residencia, la frecuencia de los desplazamientos, el viaje a un destino de Schengen, el viaje antes del mediodía y el modo de traslado al aeropuerto son factores que afectan la hora de llegada. La cuota prevista de pasajeros que llegan antes de la apertura de la facturación disminuyó al asumir que el número de pasajeros que viajan con mucha frecuencia aumenta, disminuyó cuando aumentamos la cuota de pasajeros que se encuentran alejados de la zona del aeropuerto y se mantiene estable considerando el efecto mixto de estos dos cambios

En la posterior fase de nuestra investigación, se analizaron las implicaciones futuras adicionales para los requerimientos del área de la terminal, considerando, por ejemplo, las actividades aeroportuarias. Este tipo de resultados pueden también ser utilizados para un análisis de las llegadas de los pasajeros a las áreas de facturación y de control de seguridad, con el fin de predecir los horarios punta durante el día, y planificar las acciones que correspondan para mejorar las operaciones en estos periodos.

Además, el rango de tiempo asignado a actividades no aeronáuticas puede alimentar estudios comerciales para la terminal del aeropuerto y para la propensión de los pasajeros a realizar compras dependiendo del tiempo disponible hasta la salida del vuelo. Finalmente, al enlazar estos modelos con modelos de simulación, se podría estimar cuántas personas necesitarían utilizar cada área de la terminal antes de la seguridad y comprobar las implicaciones en los requerimientos de la terminal en cada tipo de actividad.

\section{Agradecimientos}

Los autores agradecen al Aeropuerto de Lisboa Portela los permisos para llevar a cabo este trabajo. Esta investigación fue parcialmente financiada por la Fundación Nacional Portuguesa de Ciencia mediante el proyecto [SFRH/BD/51588/2011].

\section{Referencias bibliográficas}

Airport World (2015). Money matters. Recuperado de http://www.airport-world.com/ features/economics/5009-money-matters.html

Alegre, J., Mateo, S. \& Pou, L. (2009). Participation in Tourism Consumption and the Intensity of Participation: An Analysis of Their Socio-demographic and Economic Determinants. Tourism Economics 15 (3), 531-546.

Banco Mundial (2017), Data Bank. Recuperado de http://datos.bancomundial.org/ indicador/IS.AIR.PSGR 
Factores que afectan al comportamiento de los pasajeros y a

sus actividades en las terminales aeroportuarias

Barros, C.P. \& Machado, L.P. (2010). The length of stay in tourism. Annals of Tourism Research, 37 (3), 692-706.

Ben-Akiva M. \& Lerman, S. (1985), Discrete choice analysis, Cambridge: The MIT Press.

Bohl, P. (2014). The impact of airport shopping environments and dwell time on consumer spending. Budapest Management Review, 45. 11-24.

Castillo-Manzano, J.I. (2010). Determinants of commercial revenues at airports: Lessons learned from Spanish regional airports. Tourism Management, 31, 788-796.

Castillo-Manzano, J., López-Valpuesta, L. \& González-Laxe, F. (2011). The effects of the LCC boom on the urban tourism fabric: the viewpoint of tourism managers. Tourism Management, 32, 1085-1095.

Doganis, R. (1992), The airport business, New York: Routledge.

Ferrer-Rosell, B., Martínez-García, E. \& Coenders, G. (2014) Package and no-frills air carriers as moderators of length of stay. Tourism Management, 42, 114-122.

Graham, A. (2014). Managing airports: An international perspective (4th ed.). New York, NY: Routledge.

International Air Transport Association (IATA) (2004). Airport Development Reference Manual (9th Edition), Montreal-Geneva: International Air Transport Association.

International Air Transport Association (IATA) (2013). Fast travel program. Recuperado de https://www.iata.org/whatwedo/passenger/fast-travel/Documents/FastTravel-Program-Strategy-V6.pdf

Joustra, P. E. \& Van Dijk, N. M. (2001). Simulation of check-in at airports. Proceedings of Winter Simulation Conference. B. A. Peters, J. S. Smith, D. J. Medeiros, and M. W. Rohrer (eds.).

Lin, Y. H \& Chen, C. (2013), Passengers' shopping motivations and commercial activities at airports - The moderating effects of time pressure and impulse buying tendency. Tourism Management, 36, 426 - 434 .

Losada, N, Alén, E., Domínguez, T. \& Nicolau, J.L. (2016). Travel frequency of senior tourists. Tourism Management, 53, 88-95.

Ma, W. \& Yarlagadda, P. K. (2012). A micro-simulation of airport passengers with advanced traits. 28th Congress of the International Council of the Aeronautical Sciences, Brisbane: Optimage Ltd.

Park, Y. \& Ahn, S. B. (2003). Optimal assignment for check-in counters based on passenger arrival behavior at an airport. Transportation Planning and Technology. 26, Issue 5 .

Popovic, V., Kraal, B. \& Kirk, P. J. (2010) Towards airport passenger experience models. Proceedings of 7 th International Conference on Design \& Emotion, 4-7 October, Spertus Institute, Chicago, Illinois. 
Salmasi, L., Celidoni, M. \& Procidano, I. (2012). Length of stay: price and income semielasticities at different destinations in Italy. International Journal of Tourism Research, 14, 515-530.

Torres, E, Domínguez, J.S, Valdés, L. \& Aza, R. (2005). Passenger waiting time in an airport and expenditure carried out in the commercial area. Journal of Air Transport Management 11, 363-367.

Tosic, V. (1992). A review of airport passenger terminal operations analysis and modelling. Transportation Research Part A: Policy and Practice, 26, 3-26.

Van Boekhold, J., Faghri, A. \& Li, M. (2014). Evaluating security screening checkpoints for domestic flights using a general microscopic simulation model. Journal of Transportation Security, 7, 45-67.

Volkova, N. (2009). Determinants of Retail Revenue for Today's Airports. German airport performance (GAP) project. Berlin School of Economics.

World Tourism Organisation (WTO) (2014). Understanding tourism: Basic glossary. Recuperado de http://media.unwto.org/content/understanding-tourism-basicglossary 